\title{
Autoresonance control model of nonlinear dynamics of magnetization in a three-layer antiferromagnetic structure in the presence of attenuation
}

\author{
V.N. Nazarov ${ }^{1, \dagger}$, E. G. Ekomasov ${ }^{2,3}$ \\ †nazarovvn@gmail.com \\ ${ }^{1}$ Institute of Molecule and Crystal Physics Ufa Federal Research Centre of RAS, 151 Prospekt Oktyabrya, Ufa, 450075, Russia \\ ${ }^{2}$ South Ural State University (National Research University), 76 Lenin prospekt, Chelyabinsk, 454080, Russia \\ ${ }^{3}$ Bashkir State University, 32 Zaki Validi st., Ufa, 450076, Russia
}

\begin{abstract}
An autoresonance method for excitation of nonlinear breather-type oscillations of the magnetization in a three-layer antiferromagnet by applying an external alternating magnetic field is considered. The first constant of magnetic anisotropy is assumed to be a one-dimensional function of the coordinate with a local change in its value. Such dependence of the magnetic anisotropy constant on the coordinate mimics the presence of a three-layered magnetic structure in which the thick layers with the same value of anisotropy are separated by a thin layer with different value of anisotropy. The external magnetic field is represented as a resonance field and an alternating field with a small amplitude. At a constant pumping frequency, there is a small amplitude change, with solutions being constructed under the condition of parametric resonance, when the pumping frequency is equal to twice the frequency of linear ferromagnetic or antiferromagnetic resonance. Of special interest is the variable pump frequency, described by a slowly varying function of time. In such an alternating field, resonance effects may appear, leading to a significant increase in the amplitude of the breather. The main resonance equations are obtained, the analysis of which reveals the existence of solutions with elevating amplitudes. In an alternating field, the increase of amplitude of the magnetic breather is observed, whereas in the absence of the external influence, only localization of the nucleation center on the defect occurs at spatial inhomogeneity of the magnetic anisotropy. With an increase in the depth of the spatial inhomogeneity of the magnetic anisotropy, autoresonance breaks down. With a decrease in the width of the inhomogeneity region of the magnetic anisotropy or a decrease in the thickness of a thin layer in a three-layer antiferromagnet, a growing solution also exists in the alternating field, which cannot be obtained in a constant magnetic field. The effect of the initial phase of the magnetic breather on its evolution is also considered in the paper.
\end{abstract}

Keywords: magnetic solitons dynamics, three-layer ferromagnetic, autoresonance.

УДК: 537.611

\section{Авторезонансная модель управления нелинейной динамикой намагниченности в трехслойной антиферромагнитной структуре с учетом затухания в системе}

\author{
Назаров В. Н. ${ }^{1, \dagger}$, Екомасов Е. Г., \\ ${ }^{1}$ Институт физики молекул и кристаллов Уфимского федерального исследовательского центра РАН, \\ пр. Октября, 151, Уфа, 450075, Россия \\ ${ }^{2}$ Южно-Уральский государственный университет (Национальный исследовательский университет), \\ пр. Ленина, 76, Челябинск, 454080, Россия \\ ${ }^{3}$ Башкирский государственный университет, ул. Заки Валиди, 32, Уфа, 450076, Россия
}

Рассмотрен авторезонансный метод возбуждения нелинейных колебаний намагниченности бризерного типа в трехслойном антиферромагнетике внешним переменным магнитным полем. Первую константу магнитной анизотропии считаем одномерной функцией координаты с локальным изменением своего значения. Наличие подобной зависимости константы магнитной анизотропии от координаты аналогично наличию трехслойной магнитной структуры, в которой толстые слои с одинаковым значением анизотропии разделены тонким слоем с другим значением анизотропии. Внешнее магнитное поле представлено в виде резонансного поля и переменного поля с малой амплитудой. При постоянной частоте накачки существует небольшое изменение амплитуды, при этом решения строятся 
при условии параметрического резонанса, когда частота накачки равна удвоенной частоте линейного ферромагнитного или антиферромагнитного резонанса. Особый интерес представляет переменная частота накачки, описываемая медленно меняющейся функцией времени. В таком переменном поле возможно появление резонансных эффектов, приводящих к значительному росту амплитуды бризера. Получены уравнения главного резонанса, анализ которых показывает существование решений с растущими амплитудами. Когда без внешнего воздействия при определенных параметрах пространственной неоднородности магнитной анизотропии будет происходить лишь локализация зародыша на дефекте, в переменном поле наблюдается рост амплитуды магнитного бризера. С увеличением глубины пространственной неоднородности магнитной анизотропии происходит срыв авторезонанса. С уменьшением ширины области неоднородности магнитной анизотропии или уменьшением толщины тонкого слоя в трехслойном антиферромагнетике в переменном поле также имеет место растущее решение, которое не удается получить в постоянном магнитном поле. В работе также показано влияние начальной фазы магнитного бризера на его эволюцию.

Ключевые слова: динамика магнитных солитонов, трехслойный ферромагнетик, авторезонанс.

\section{1. Введение}

Нелинейному ферромагнитному резонансу посвящено много работ как экспериментального, так и теоретического характера (см., например, [1-5]). В последнее время внимание исследователей привлекает возможность возбуждения в малых частицах, тонких ферромагнитных пленках высокоамплитудной прецессии намагниченности и их перемагничивания высокочастотными полями (см., например, [6-9]). Во многих работах высокоамплитудная прецессия намагниченности исследуется в случае не малых амплитуд переменного поля. Кроме того, в работах часто не ставится проблема управления нелинейной динамикой намагниченности в реальном времени. Развитие теории управления нелинейной динамической системой в условиях резонанса является весьма важным для технических приложений [10]. В этом контексте выявление эффекта “захвата" нелинейных колебаний намагниченности магнитными полями небольшой амплитуды и определение условий авторезонансной генерации колебаний намагниченности в условиях автофазировки в феррои антиферромагнетиках со слабым ферромагнетизмом при наличии магнитных дефектов является интересной и актуальной задачей. Для решения подобной задачи для уравнения Ландау-Лифшица-Гильберта, описывающего динамику намагниченности, необходимо найти решения, траектория которых стартует вблизи устойчивого положения равновесия системы и со временем уходит на достаточное удаление от этого состояния, притом, что радиочастотные поля остаются малыми. Такое поведение намагниченности обусловливается резонансными эффектами в конденсированных средах и в нелинейных системах ассоциируется с термином автофазировки. Под автофазировкой в физике понимается явление автоматической подстройки фаз слабо связанных осцилляторов. При значительном изменении амплитуды колебаний это явление принято называть авторезонансом. Явление авторезонанса было открыто В.И. Векслером в 1944 году при создании ускорителей релятивистских частиц. Существенным условием авторезонансной генерации нелинейных колебаний является медленное изменение частоты радиочастотного поля, которая в начальный момент близка к резонансной. Систематическое исследование математических моделей авторезонанса, представимых в форме дифференциаль- ных уравнений, началось с работы Меерсона и Фридлянда [11]. Известно, что авторезонанс обнаруживается в колебательных системах различной природы [11-13]. Явление авторезонанса, описываемое нелинейными дифференциальными уравнениями в частных производных (см., например, [14-15]) исследовано достаточно слабо. Приложения теории авторезонанса к исследованию динамики нелинейных образований в средах с магнитным упорядочением при наличии внешнего магнитного поля, дефектов и диссипации сделаны в работах [16-21]. Найдены условия возникновения авторезонанса, при котором происходит значительный рост амплитуды локальных магнитных неоднородностей бризерного и солитонного типа. Разработана солитонная модель неоднородного перемагничивания магнетиков, основанная на использовании законов изменения энергии и числа спиновых отклонений при наличии внешних возмущений и диссипации. Найдены методы теоретического исследования влияния магнитных дефектов на динамику зародыша новой фазы. Рассмотрены некоторые задачи об авторезонансном возбуждении болшеамплитудных колебаний в нелинейной системе. Особый интерес представляет изучение контролируемых динамических условий, в которых высокие углы прецессии намагниченности могут быть достигнуты применением полей достаточно малой амплитуды [22].

Эффект авторезонанса является чисто нелинейным явлением и обусловлен зависимостью собственной частоты колебаний от энергии. Пока он хорошо изучен только для одномерных систем при отсутствии диссипации. В реальных материалах всегда присутствует диссипация, и содержатся различного рода неоднородности, обусловленные дефектами решетки, внешними воздействиями или многослойностью образца. Развитие теории авторезонансного возбуждения неоднородных колебаний намагниченности с большой амплитудой может позволить разработать эффективный метод управления зародышеобразованием и перемагничиванием. Выявление условий, при которых возникает авторезонанс в реальных ферро- и антиферромагнетиках и является целью данной статьи. В данной работе рассматривается авторезонансное параметрическое возбуждение магнитного бризера в трехслойном антиферромагнетике полями переменной частоты и малой амплитуды. Считается также, что диссипация слабая и частота является медленной функцией времени. 


\section{2. Основные уравнения и результаты}

Рассмотрим случай антиферромагнетика ромбической симметрии. Плотность функции Лагранжа $L$ и диссипативную функция Рэлея $R$, как функции от вектора антиферромагнетизма $\boldsymbol{l}$, имеют вид $[23,24]$ :

$$
\begin{gathered}
L=\frac{\chi_{\perp}}{2 \gamma^{2}} \dot{\boldsymbol{l}}^{2}-F+M_{0} l_{x} H_{z}, \quad R=\frac{\alpha M_{0}}{2 \gamma} \dot{\boldsymbol{l}}^{2}, \\
F=\frac{1}{2} A(\nabla \boldsymbol{l})^{2}-\frac{1}{2} K_{a b} l_{x}^{2}-\frac{1}{2} K_{b c} l_{z}^{2}+ \\
+\frac{1}{4}\left(K_{2}^{(11)} l_{x}^{4}+K_{2}^{(13)} l_{x}^{2} l_{z}^{2}+K_{2}^{(33)} l_{z}^{4}\right) .
\end{gathered}
$$

Здесь $\chi_{\perp}-$ антиферромагнитная восприимчивость, $\gamma-$ гиромагнитное отношение, $\alpha-$ параметр затухания, $M_{0}$ - намагниченность насыщения, $A-$ константа неоднородного обменного взаимодействия, $K_{a b}, K_{b c}, K_{2}^{(i j)}-$ константы магнитной анизотропии, $H_{z}-$ внешнее магнитное поле вдоль $c$-оси. В ромбическом кристалле при $K_{b c}<0$ спонтанные спин-переориентационные фазовые переходы происходят путем поворота вектора антиферромагнетизма $\boldsymbol{l}$ в $a b$-плоскости $[23,25]$. При этом плотность энергии эффективной магнитной анизотропии можно представить в виде:

$$
F_{a n}=\text { const }+\frac{1}{2} K_{1} \sin ^{2} \theta+\frac{1}{2} K_{2} \sin ^{4} \theta
$$

где $\theta$ - угол между $\boldsymbol{y} \| b$-оси и вектором $\boldsymbol{l}$ в $a b$-плоскости, $K_{1}=-K_{a b}, K_{2}=K_{2}^{(11)} / 2$. Как известно [25], при $K_{1} \geq 0$ устойчива фаза $G_{y}$, где $l \| b$-оси $(\theta=0, \pi)$, а при $K_{1}+2 K_{2} \leq 0-$ фаза $G_{x} F_{z}$, где $\boldsymbol{l}\|\boldsymbol{x}\|$-оси $(\theta=\pi / 2,3 \pi / 2)$. В случае отрицательной второй константы магнитной анизотропии, то есть $K_{2}<0$, при $K_{1}+K_{2}=0$ имеет место фазовый переход первого рода между антиферромагнитной $\left(G_{y}\right)$ и слабоферромагнитной $\left(G_{x} F_{z}\right)$ фазами.

Первую константу магнитной анизотропии считаем одномерной функцией координаты $\xi=y / \delta_{0}$ :

$$
\begin{gathered}
K_{1}(\xi)=K_{1}[1-k f(\xi)], \\
f(\xi)=\Theta(\xi+d / 2)-\Theta(\xi-d / 2), \\
\Theta(z)= \begin{cases}1, & z \geq 0, \\
0, & z<0,\end{cases}
\end{gathered}
$$

где $f(\xi)$ характеризует локальное изменение магнитной анизотропии, $k=\Delta K_{1} / K_{1}, \Delta K_{1}>0-$ величина локального изменения константы магнитной анизотропии, $\delta_{0}=\left(A /\left|K_{2}\right|\right)^{1 / 2}-$ характерный размер 90-градусной межфазной границы, $d=D / \delta_{0}, D-$ ширина области с пониженной анизотропией. Отметим, что подобный вид зависимости константы магнитной анизотропии от координаты достаточно часто используется при решении подобных задач (см., например, [26-32]). Наличие подобной зависимости константы магнитной анизотропии от координаты аналогично наличию трехслойной магнитной структуры, где широкие слои с одинаковым значением анизотропии разделены узким слоем со значением анизотропии отличным от его значения в толстых слоях. Так же по общепринятой терминологии [26] можно считать, что в работе рассматривается уединенный дефект магнитной анизотропии, что вполне оправдано, так как период магнитных неоднородностей в образце (порядка 20-30 $\mu \mathrm{m}$ ) значительно превосходит размер зародыша новой фазы. Следует заметить, что при изменении, например, химического состава кристалла в слоях возможно также и изменение константы обменного взаимодействия, но его влияние на эволюцию зародыша новой фазы (см., например, [28-31]) часто приводит к перенормировке критических значений параметров материала и в данной работе мы им будем пренебрегать.

Из уравнения Лагранжа с учетом (1) - (2) можно получить следующее уравнение, описывающее динамику одномерных магнитных неоднородностей:

$$
\begin{aligned}
& \psi_{\tau \tau}-\psi_{\xi \xi}+\sin \psi=-\beta \psi_{\tau}- \\
&-2[g-(1+g) k f(\xi)] \sin \frac{\psi}{2}+4 h \cos \frac{\psi}{2} .
\end{aligned}
$$

Здесь $\psi=4 \theta, \tau=\left(c / \delta_{0}\right) t, c=\gamma\left(A / \chi_{\perp}\right)^{1 / 2}-$ предельная скорость межфазных стенок, совпадающая с минимальной фазовой скоростью спиновых волн на линейном участке их закона дисперсии; $\beta=\alpha M_{0} /\left(\left|K_{2}\right| \chi_{\perp}\right)^{1 / 2}$. Отметим, что подобным уравнением (3) можно описывать динамику солитонов и в других системах [33].

В отсутствие затухания, внешнего магнитного поля и дефектов с граничными условиями $\psi(|\xi| \rightarrow \infty)=0$, $\psi_{\xi}(|\xi| \rightarrow \infty)=0$ уравнение (3) будем иметь двухсолитонное решение вида с параметрами $\Omega$ и $р$ солитона

$$
\operatorname{tg}^{2} \frac{\psi}{4}=\frac{1-\Omega}{\Omega+p^{2}} \cdot \frac{1}{\operatorname{ch}^{2}(\xi \sqrt{1-\Omega})} .
$$

При $\Omega+p^{2}>0$ это решение описывает динамическую 0 -градусную стенку. Когда $|g| \ll 1, k \ll 1, \beta \ll 1$ решение (4) можно рассматривать как приближенное решение (3), где $\Omega=\Omega(\tau), p=p(\tau)$ являются неопределенными функциями времени. Уравнения для $\Omega$ и $p$ в адиабатическом приближении можно получить из законов изменения энергии и числа спиновых отклонений:

$$
\begin{aligned}
& \Omega_{\tau}=2 \frac{p(\beta p-g)(1-\Omega)}{p^{2}+1}(1+\Gamma(\Omega, p))+ \\
&+ 2 \frac{p k(g+1)(1-\Omega)}{p^{2}+1} I(\Omega, p, d)+2 p a h J(\Omega, p) \\
& p_{\tau}=\Omega+p^{2}-\beta p+g-k \frac{(g+1) I(\Omega, p, d)}{1+\Gamma(\Omega, p)}- \\
&-\frac{a h\left(p^{2}+1\right)}{(1-\Omega)(1+\Gamma(\Omega, p))} J(\Omega, p)
\end{aligned}
$$

где

$$
\begin{gathered}
I(\Omega, p, d)=\frac{\operatorname{th}(r)}{1+a^{2}-a^{2} \operatorname{th}^{2}(r)}+\frac{1}{2 a \sqrt{1+a^{2}}} \operatorname{Arth}\left(\frac{\sqrt{1+a^{2}}}{a \operatorname{th}(r)}\right), \\
\Gamma(\Omega, p)=\frac{1}{a \sqrt{1+a^{2}}} \operatorname{Arth}\left(\frac{a}{\sqrt{1+a^{2}}}\right), \\
r=\frac{d}{2} \sqrt{1-\Omega}, \quad a=\left(\frac{1-\Omega}{\Omega+p^{2}}\right)^{1 / 2}, \\
J(\Omega, p)=\int_{-\infty}^{\infty} \frac{\operatorname{ch}^{2} x d x}{\left(\operatorname{ch}^{2} x+a^{2}\right)^{3 / 2}} .
\end{gathered}
$$

Полагая

$$
\Omega=\omega^{2}, \quad p=-\omega \operatorname{ctg} \chi,
$$


систему уравнений (5) можно привести к виду, полученному на основе метода обратной задачи рассеяния:

$$
\begin{aligned}
\frac{d \omega}{d \tau}= & \frac{\operatorname{ctg} \chi\left(1-\omega^{2}\right)}{1+\omega^{2} \operatorname{ctg}^{2} \chi}((\beta \omega \operatorname{ctg} \chi+g)(1+\Gamma)-k(g+1) N)- \\
& -\cos \chi \frac{\sqrt{1-\omega^{2}}}{\omega} h J \\
\frac{d \chi}{d \tau}= & +\left(\beta \omega \operatorname{ctg} \chi+g-k \frac{g+1}{1+\Gamma} \Phi\right) \frac{1+\Gamma \cos ^{2} \chi\left(1-\omega^{2}\right)}{\omega\left(1+\omega^{2} \operatorname{ctg}^{2} \chi\right)}- \\
& -\left(\operatorname{ctg}^{2} \chi \sin \chi\left(1-\omega^{2}\right)+\frac{1+\omega^{2} \operatorname{ctg}^{2} \chi}{\Gamma}\right) \frac{h J}{\omega \sqrt{1-\omega^{2}}},
\end{aligned}
$$

где

$$
\begin{gathered}
\Gamma=\frac{\omega \operatorname{arcsh}(A \sin \chi / \omega)}{A \sin \chi \sqrt{1+(A \sin \chi / \omega)^{2}}}, \\
N=\frac{\operatorname{th} r}{1+\left(1-\operatorname{th}^{2} r\right)(A \sin \chi / \omega)^{2}}+ \\
+\frac{\omega /(A \sin \chi)}{2 \sqrt{1+(A \sin \chi / \omega)^{2}}} \ln \left|\frac{A \sin \chi \operatorname{th} r+\sqrt{\omega^{2}+A^{2} \sin ^{2} \chi}}{A \sin \chi \operatorname{th} r-\sqrt{\omega^{2}+A^{2} \sin ^{2} \chi}}\right| .
\end{gathered}
$$

Анализ уравнений (5) был проведен лишь для сравнительно простой ситуации, когда внешнее поле либо отсутствует [34], либо оно постоянно $h=h_{0}$ [35]. В постоянном магнитном поле рост зародыша новой фазы будет увеличиваться, когда ширина начального зародыша сравнима с шириной $d$ области пониженной анизотропии. В случае, когда начальная ширина зародыша больше ширины $d$, роста зародыша добиться не удается, в постоянном поле он может только локализоваться на дефекте. Никакого роста амплитуды в этих условиях получить нельзя.

В случае переменного внешнего поля $h(t)$ возможно появление резонансных эффектов, которые приводят к более значительному изменению амплитуды. При постоянной частоте накачки и в отсутствии диссипации возможно изменение амплитуды $A(t)$ на величину порядка $O(\sqrt{h})$. Такого типа решения строятся при условии параметрического резонанса, когда частоты связаны соотношением $2 \omega=2\left(1-A_{0}^{2}\right)^{1 / 2}=\omega^{\prime}$, где $\omega^{\prime}-$ частота внешнего воздействия. Достичь изменений амплитуды на величину порядка единицы при малой амплитуде накачки на этом пути невозможно: при больших изменениях $A(t)$ нарушается резонансное условие из-за зависимости собственной частоты от амплитуды $\omega=\left(1-A^{2}\right)^{1 / 2}[16]$.

Рассмотрим переменную частоту накачки $\omega^{\prime}-\mu \tau$ $(\mu>0)$. Внешнее магнитное поле представим в виде резонансного поля $h_{0}$ и переменного поля с амплитудой $h_{1}$ :

$$
h=h_{0}+h_{1} \cos \left(\omega^{\prime} \tau-\mu \tau^{2} / 2\right),
$$

частота которого $\omega^{\prime}-\mu \tau$ медленно меняется при $|\mu|=$ const $\ll 1$. Здесь при малых $\mu, h_{1}$ обнаруживаются приближенный решения со значительным изменением амплитуды. Эволюция во времени квадрата амплитуды $A^{2}(t)$ на таких решениях определяется из резонансного условия:

$$
2\left(1-A^{2}\right)^{1 / 2}=\omega^{\prime}-\mu \tau
$$

Это соотношение интерпретируется как захват системы в параметрический резонанс.

Введем новые переменные $\quad \omega\left(\tau-\tau_{0}\right)=\Phi(\tau)$,
$A=\left(1-\omega^{2}\right)^{1 / 2}$. Тогда решение невозмущенного уравнения Ландау - Лифшица:

$$
\operatorname{tg} \frac{\Psi}{4}=\frac{A}{\omega} \frac{\sin \chi}{\cosh A \xi} .
$$

Для получения уравнений главного резонанса воспользуемся анзацем вида:

$$
A=\sqrt{\varepsilon R}, \varepsilon R \ll 1, \chi=\left(\omega^{\prime} \tau-\mu \tau^{2} / 2+\Psi(\varepsilon \tau)\right) / 2 .
$$

Здесь $R(\varepsilon \tau)$ является медленно меняющейся функцией времени, $0<\varepsilon<<1$. Уравнения для функций $R(\tau), \Psi(\tau)$, которые принято называть уравнениями главного резонанca, получаются путем усреднения. При выполнении резонансного условия $2 \omega(0)=\omega^{\prime}$ эти уравнения имеют вид:

$$
\begin{gathered}
\frac{d R}{d \tau^{\prime}}=-\frac{2 R \beta}{\varepsilon}-\frac{R h_{1}}{\varepsilon} \sin \psi, \\
\frac{d \Psi}{d \tau^{\prime}}=-R-\frac{h_{1}}{\varepsilon} \cos \psi+\frac{\mu}{\varepsilon^{2}} \tau^{\prime}- \\
-\frac{2 k(g+1)}{\varepsilon} b_{1}+\frac{2\left(g+2 h_{0}\right)}{\varepsilon} b_{2},
\end{gathered}
$$

где

$$
\begin{gathered}
b_{1}=\frac{1}{2 \pi} \int_{0}^{2 \pi} \frac{L \Phi \sin ^{2} \eta}{1+\Gamma} d \eta, \quad b_{2}=\frac{1}{2 \pi} \int_{0}^{2 \pi} L \sin ^{2} \eta d \eta \\
L=\frac{1+A^{2} \cos ^{2} \eta \cdot \Gamma}{1-A^{2} \cos ^{2} \eta}
\end{gathered}
$$

Анализ системы уравнений главного резонанса в случае $h_{0}=0$ при следующих соотношениях малых параметров $\left|h_{1}\right| / \varepsilon=1, \mu / \varepsilon^{2} \approx 1, \beta / \varepsilon \leq O(1)$ показывает существование решений с растущими амплитудами (Рис. 1). Видно, что для ширины $d=8$ и глубины $k=0.08$ наблюдается рост амплитуды в переменном поле, в то время как без внешнего воздействия будет происходить лишь локализация зародыша на дефекте [34]. С увеличением параметра затухания колебательное движение становится слабо заметно и будет происходить почти монотонное увеличение амплитуды (кривая 2, Рис. 1). С увеличением глубины неоднородности анизотропии будет наблюдаться не растущее решение (кривая 3, Рис. 1). С уменьшением ширины потенциальной ямы можно получить растущее решение (Рис. 2 , кривая 1,2 ) и срыв авторезонанса (Рис. 2, кривая 3) при большем затухании. При таких параметрах (малой ширины $d$ ) в отсутствии внешнего воздействия начальный зародыш превращается в затухающий бризер [34], либо в постоянном поле он локализуется на дефекте [35]. В переменном поле, как показывает анализ уравнений (7), можно получить растущие решения (Рис. 2).

Когда ширина $d$ области с пониженной анизотропией больше ширины начального зародыша, в отсутствии внешнего воздействия происходит рост зародыша с образованием домена новой фазы, межфазные стенки выходят за пределы такой области, не совершая колебаний, и двигаются в противоположных направления с постоянной скоростью [34]. Подобное разбегание стенок описана также в [36]. Из Рис. 3 видно, что в переменном поле существует также и не растущее решение.

Заметим, что растущие и не растущие решения зависят также от начальной фазы $\Psi(0)$, меняя значение которой можно получить различную эволюцию магнитного бризера при одних параметрах (Рис. 4, кривые 2 и 3). 


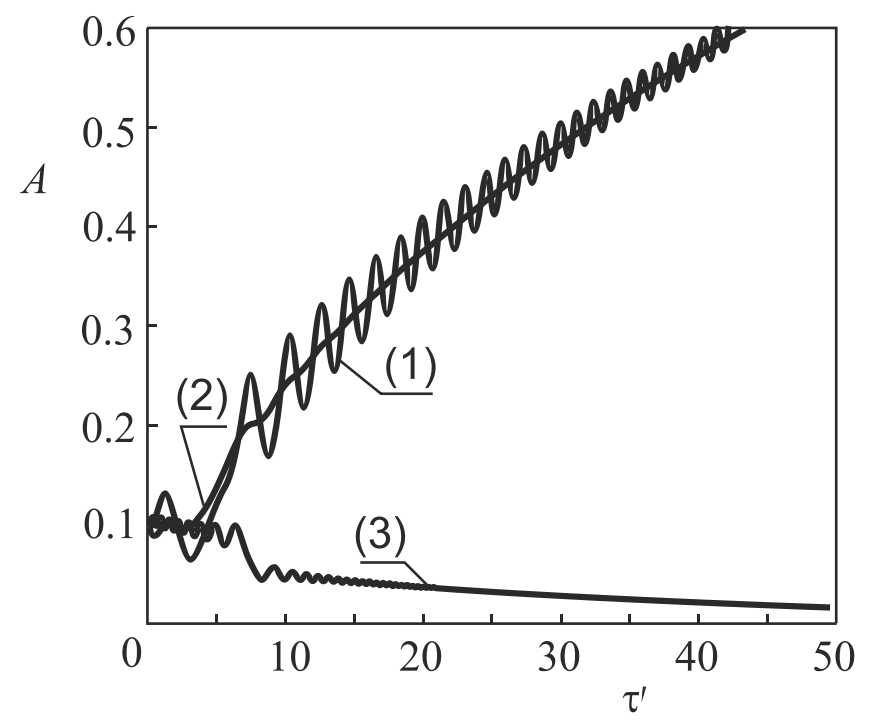

Рис. 1. Растущие решения уравнений главного резонанса (зависимость амплитуды магнитного бризера от времени) при $k=0.08, \beta=0.0003$ (кривая 1), $k=0.08, \beta=0.003$ (кривая 2) и не растущее решение при $k=0.3, \beta=0.0003$ (кривая 3). Здесь $g=0.01, d=8, h_{1}=0.01, R(0)=1, \psi(0)=\pi / 4$.

Fig. 1. The increasing solutions of the main resonance equations (the dependence of the amplitude of the magnetic breather on time) corresponding to $k=0.08, \beta=0.0003$ (curve 1), $k=0.08$, $\beta=0.003$ (curve 2) and the non-increasing solution observed for $k=0.3, \beta=0.0003$ (curve 3). Here $g=0.01, d=8, h_{1}=0.01, R(0)=1$, $\psi(0)=\pi / 4$.

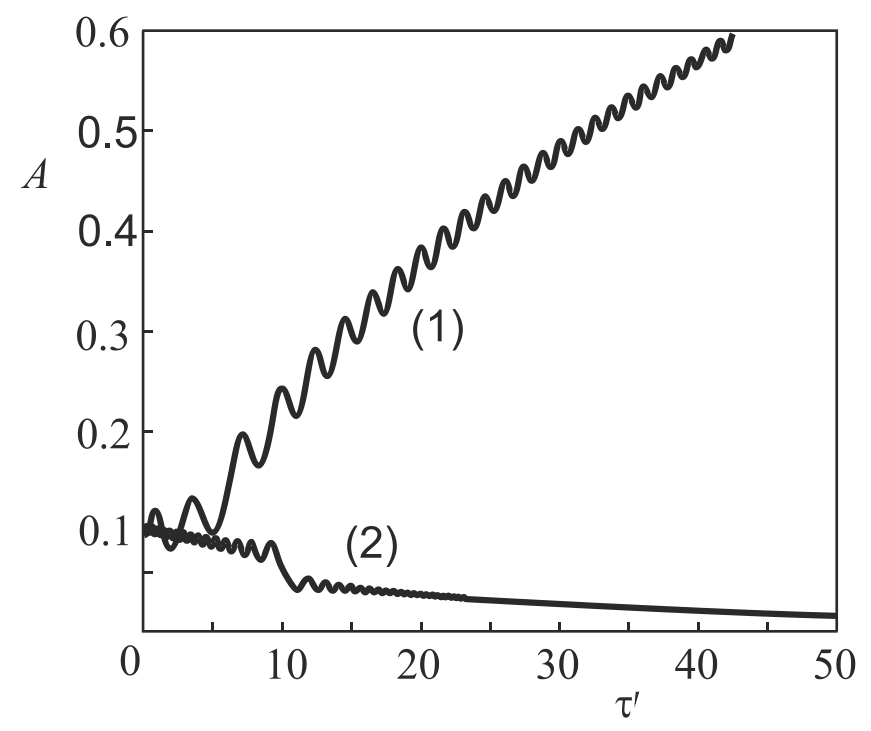

Рис. 3. Растущее решение уравнений главного резонанса при $k=0.08$ (кривая 1) и не растущее решение при $k=0.3$ (кривая 2). Здесь $g=0.01, d=12, \beta=0.0003, R(0)=1, \psi(0)=\pi / 4$.

Fig. 3. The increasing solution of the main resonance equations (the dependence of the amplitude of the magnetic breather on time) corresponding to $k=0.08$ (curve 1) and the non-increasing solution observed for $k=0.3$ (curve 2). Here $g=0.01, d=12, \beta=0.0003$, $R(0)=1, \psi(0)=\pi / 4$.

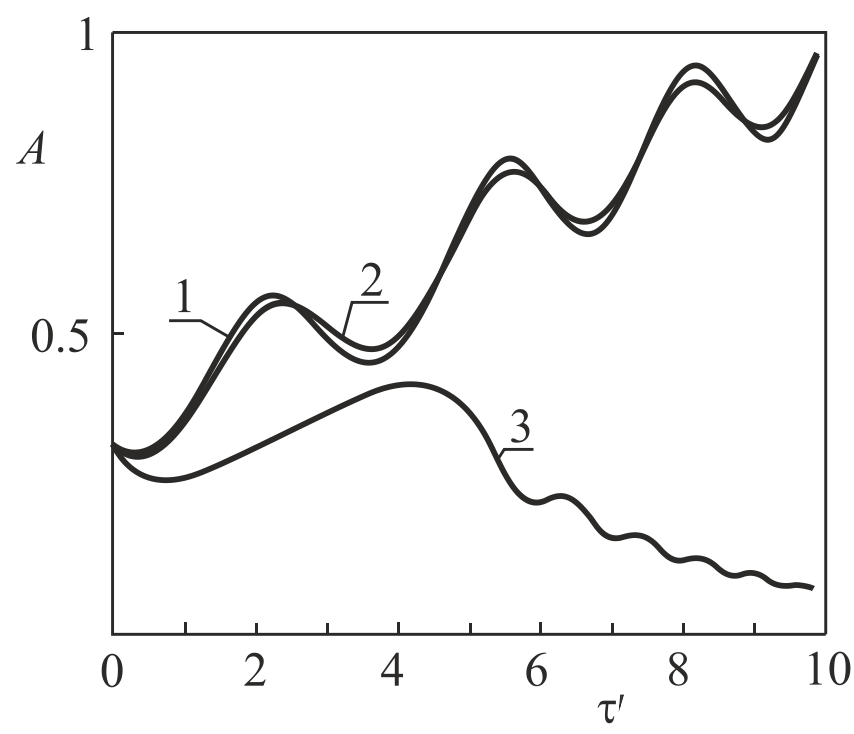

Рис. 2. Растущие решения уравнений главного резонанса при $\beta=0.0003$ (кривая 1), $\beta=0.003$ (кривая 2) и не растущее решение при $\beta=0.03$ (кривая 3). Здесь $k=0.3, g=0.01, d=2$, $h_{1}=0.1, R(0)=1, \psi(0)=\pi / 4$.

Fig. 2. The increasing solutions of the main resonance equations (the dependence of the amplitude of the magnetic breather on time) corresponding to $\beta=0.0003$ (curve 1 ), $\beta=0.003$ (curve 2) and the non-increasing solution observed for $\beta=0.03$ (curve 3 ). Here $k=0.3, g=0.01, d=2, h_{1}=0.1, R(0)=1, \psi(0)=\pi / 4$.

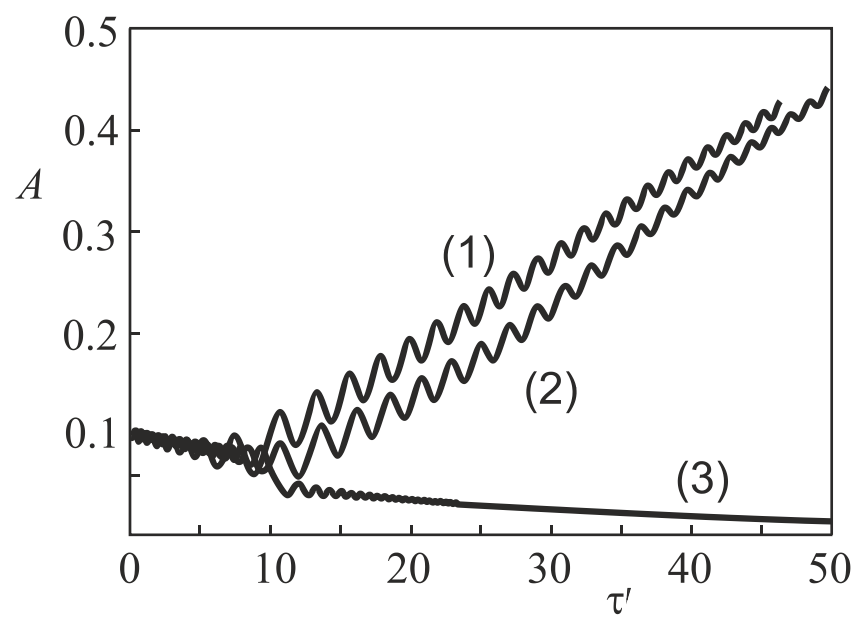

Рис. 4. Растущие решения уравнений главного резонанса при $d=8$ (кривая 1 ) и $d=12$ (кривая 2) с начальными условиями $\psi(0)=\pi / 2$, и не растущее решение при $d=3$ (кривая 3) с другой начальной фазой $\psi(0)=\pi / 4$. Здесь $g=0.01, k=0.3, \beta=0.0003$, $R(0)=1$.

Fig. 4. The increasing solutions of the principal resonance equations corresponding to $d=8$ (curve 1) and $d=12$ (curve 2) with initial conditions $\psi(0)=\pi / 2$, and non-increasing solution corresponding to $d=8$ (curve 3 ) with different initial phase $\psi(0)=\pi / 4$. Here $g=0.01, k=0.3, \beta=0.0003, R(0)=1$. 


\section{3. Заключение}

В работе рассмотрена динамика локализованных магнитных неоднородностей в трехслойном антиферромагнетике в переменном внешнем магнитном поле малой амплитуды. Показано, что при различных значениях магнитных параметров в слоях и величины затухании могут наблюдаться растущие и не растущие по амплитуде локализованные магнитные неоднородности бризерного типа. Получены уравнения главного резонанса, из анализа которых следует возможность авторезонансного параметрического возбуждения магнитного бризера, что не удается получить для случая постоянного магнитного поля. Обнаружено влияние начальной фазы магнитного бризера на его эволюцию.

Благодарности/Acknowledgements. Cтатья выполнена при поддержке Правительства РФ (Постановление № 211 om 16.03.2013 г., соглашение № 02. A03.21.0011)./The work was supported by Act 211 Government of the Russian Federation, contract № 02. A03.21.0011.

\section{Литература/References}

1. Ya.A. Monosov. Nonlinear ferromagnetic resonance. Moscow, Nauka (1971)210p. (in Russian) [Я. А. Моносов. Нелинейный ферромагнитный резонанс. Москва, Наука (1971) 210 c.]

2. A.G. Gurevich, G.A. Melkov. Magnetic oscillations and waves. Moscow, Main Editorial for Literature on Physics and Mathematics (1994) 464 p. (in Russian) [А.Г. Гуревич, Г.А. Мелков. Магнитные колебания и волны. Москва, Физматлит (1994) 464 с.]

3. G. S. Patrin, N. V. Volkov, N. V. Fedoseeva, E. M. Nikolaev. Pis'ma Zh. Eksp. Teor. Fiz. 57(3), 189 (1993). (in Russian) [Г. С. Патрин, Н.В. Волков, Н. В. Федосеева, Е. М. Николаев. Письма в ЖЭТФ. 57(3), 183 (1993).]

4. G.S. Patrin, N.V. Volkov. Pis'ma Zh. Eksp. Teor. Fiz. 64(12), 841 (1996). (in Russian) [Г.С. Патрин, Н.В. Волков. Письма в ЖЭТФ. 64(12), 841 (1996).] DOI: $10.1134 / 1.567241$

5. G. S. Patrin, N. V. Volkov, I. V. Prokhorova. Phys. Solid State. 46(10), 1891 (2004). (in Russian) [Патрин Г.C., Волков Н.В., Прохорова И.В. ФТТ. 46(10), 1828 (2004).] DOI: 10.1134/1.1809426

6. C. Thirion, W. Wernsdorfer, D. Mailly. Nature materials. 2(8), 524 (2003). DOI: 10.1038/nmat946

7. D. I. Sementsov, A. M. Shutyi. Phys. Usp. 50, 793 (2007). (in Russian) [Д.И. Семенцов, А. М. Шутый. УФН. 177(8), 831 (2007).] DOI: 10.1070/PU2007v050n08ABEH006147

8. L. N. Kotov, L.S. Nosov. Technical Physics. 50(10), 1305 (2005). (in Russian) [Л.Н. Котов, Л.С. Носов. ЖТФ. 75(10), 55 (2005).] DOI: 10.1134/1.2103276

9. S.I. Denisov, T.V. Lyutyy, P. Hänggi,
K. N. Trohidou. Phys. Rev. B. 74(10), 104406 (2006). DOI: 10.1103/PhysRevB.74.104406

10. A.L. Fradkov. Phys. Usp. 48, 103 (2005). (in Russian) [А.Л. Фрадков. УФН. 175(2), 113 (2005).] DOI: 10.1070/PU2005v048n02ABEH002047

11. B. Meerson, L. Friedland. Phys. Rev. A. 41(9), 5233 (1990). DOI: 10.1103/PhysRevA.41.5233

12. J. Fajans, L. Friedland. Am. J. Phys. 69(10), 1096 (2001). DOI: $10.1119 / 1.1389278$

13. L. A. Kalyakin. J. Math. Sci. 125(5), 658 (2005). (in Russian) [Калякин Л. А. Современная математика и ее приложения. 5, 79 (2003).] DOI: 10.1007/PL00021950

14. L. Friedland, A. G. Shagalov. Phys. Rev. E. 71, 036206 (2005). DOI: 10.1103/PhysRevE.71.036206

15. L. Friedland, A. G. Shagalov. Phys. Rev. E. 73, 0666122006 (2006). DOI: 10.1103/PhysRevE.73.066612

16. R.N. Garifullin, L.A. Kalyakin, M.A. Shamsutdinov. Comput. Math. and Math. Phys. 47, 1158 (2007). (in Russian) [Р.Н. Гарифуллин, Л.А. Калякин, М. А. Шамсутдинов. Ж. вычисл. матем. и матем. физ. 47(7), 1208 (2007).] DOI: 10.1134/S0965542507070081

17. L.A. Kalyakin, M.A. Shamsutdinov. Theor. Math. Phys. 160, 960 (2009). (in Russian) [Л.А. Калякин, М.А. Шамсутдинов. ТМФ. 160(1), 102 (2009).] DOI: $10.1007 / \mathrm{s} 11232-009-0086-3$

18. V.N. Nazarov, L.A. Kalyakin, M.A. Shamsutdinov. Solid State Phenomena. 168-169, 81 (2011). DOI: 10.4028/www.scientific.net/SSP.168-169.81

19. S. V. Batalov, A.G. Shagalov. Phys. Metals Metallogr. 109(1), 1 (2010). (in Russian) [C.B. Баталов, А.Г. Шагалов. ФММ. 109(1), 3 (2010).] DOI: 10.1134/S0031918X10010011

20. S. V. Batalov, A.G. Shagalov. Phys. Metals Metallogr. 114(2), 103 (2013). (in Russian) [C.В. Баталов, А. Г. Шагалов. ФММ. 114(2), 115 (2013).] DOI: $10.1134 /$ S0031918X13020038

21. I. Kayumov, E. Shikhovtseva, V. Nazarov. Letters on materials. 6(3), 173 (2016). (in Russian) [И.Р. Каюмов, Е.С. Шиховцева, В.Н. Назаров.

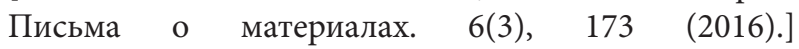
DOI: $10.22226 / 2410-3535-2016-3-173-178$

22. L. A. Kalyakin. Russian Math. Surveys. 63(5), 791 (2008). (in Russian) [Л.А. Калякин. УМН. 63(5), 3 (2008).] DOI: 10.1070/RM2008v063n05ABEH004560

23. M. A. Shamsutdinov, I. Yu. Lomakina, V.N. Nazarov, A.T. Kharisov, D.M. Shamsutdinov. Ferro- and Antiferromagnetodynamics. Nonlinear Oscillations, Waves, and Solitons. Moscow, Nauka (2009) 456 p. (in Russian) [M.А. Шамсутдинов, И.Ю. Ломакина, В.Н. Назаров, А.Т. Харисов, Д. М. Шамсутдинов. Ферро- и антиферромагнитодинамика. Нелинейные колебания, волны и солитоны. Москва, Наука (2009) 456 c.]

24. V.N. Nazarov, R.R. Shafeev, M.A. Shamsutdinov, 
I. Yu. Lomakina. Phys. Solid State. 54, 298 (2012). (in Russian) [B.Н. Назаров, Р.P. Шафеев, М. А. Шамсутдинов, И. Ю. Ломакина. ФТТ. 54(2), 282 (2012).] DOI: 10.1134/S1063783412020205

25. K. P. Belov, A. K. Zvezdin, A. M. Kadomtseva, R. Z. Levitin. Orientational transitions in rare-earth magnets. Moscow, Nauka (1979) 317 p. (in Russian) [К.П. Белов, А.К. Звездин, А.М. Кадомцева, Р.3. Левитин. Ориентационные переходы в редкоземельных магнетиках. Москва, Наука (1979) 317 с.]

26. V.V. Plavskii, M.A. Shamsutdinov, E. G. Ekomasov, A. G. Davletbaev. Phys. Metals Metallogr. 75, 589 (1993). (in Russian) [В.В. Плавский, М.А. Шамсутдинов, Е. Г. Екомасов, А. Г. Давлетбаев. ФММ. 75(6), 26 (1993).]

27. E.G. Ekomasov, S.A. Azamatov, R.R. Murtazin, A.M. Gumerov, A.D. Davletshina. Bull. Russ. Acad. Sci. Phys. 74(10), 1459 (2010). (in Russian) [Е. Г. Екомасов, Ш.А. Азаматов, Р.Р. Муртазин, А.М. Гумеров, А.Д. Давлетшина. Известия РАН. Серия физическая. 74(10), 1520 (2010).] DOI: $10.3103 /$ S1062873810100412

28. E.G. Ekomasov, R.R. Murtazin, S.A. Azamatov. Phys. Solid State. 54(8), 1584 (2012). (in Russian) [Е. Г. Екомасов, Р. Р. Муртазин, Ш. А. Азаматов. ФТТ. 54(8), 1487 (2012).] DOI: 10.1134/S1063783412080112
29. E. G. Ekomasov, R.R. Murtazin, O.B. Bogomazova, A.M. Gumerov. JMMM. 339, 133 (2013). DOI: 10.1016/j.jmmm.2013.02.042

30. E. G. Ekomasov, R.R. Murtazin, V.N. Nazarov. Phys. Metals Metallogr. 115(2), 117 (2014). (in Russian) [Е. Г. Екомасов, Р. Р. Муртазин, В.Н. Назаров. ФММ. 115(2), 125 (2014).] DOI: 10.1134/S0031918X14020069

31. E. Ekomasov, R. Murtazin, O. Bogomazova, V. Nazarov. Materials Science Forum. 845, 195 (2016). DOI: 10.4028/www.scientific.net/MSF.845.195

32. E. G. Ekomasov, M. A. Shabalin. Phys. Metals Metallogr. 101(1), 48 (2006). DOI: 10.1134/S0031918X06130151

33. E. S. Shikhovtseva, V.N. Nazarov. Eur. Biophys. J. 47, 69 (2018). DOI: 10.1007/s00249-017-1230-x

34. V.N. Nazarov, R.R. Shafeev, M.A. Shamsutdinov. I. Yu. Lomakina. Phys. Solid State. 54, 298 (2012). (in Russian) [B.Н. Назаров, P.P. Шафеев, М. А. Шамсутдинов, И. Ю. Ломакина. ФТТ. 54(2), 282 (2012).] DOI: 10.1134/S1063783412020205

35. V. Nazarov, R. Shafeev. Mod. Phys. Lett. B. 26. 1250183 (2012). DOI: 10.1142/S0217984912501837

36. V. Nazarov, E. Ekomasov. Letters on materials. 6(4), 257 (2016). (in Russian) [B.Н. Назаров, Е. Г. Екомасов. Письма о материалах. 6(4), 257 (2016).] DOI: 10.22226/2410-3535-2016-4-257-261 